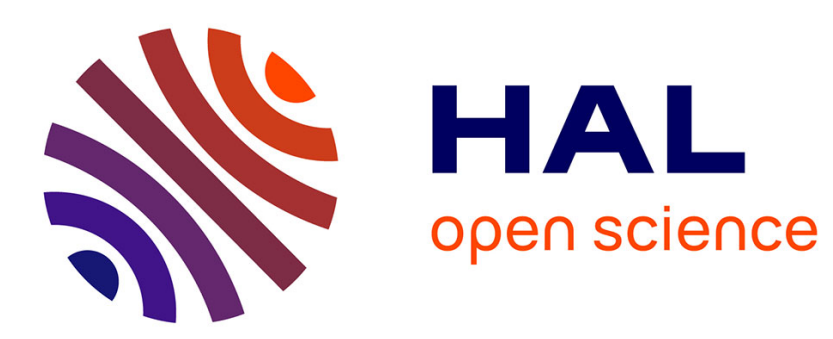

\title{
Le témoignage de l'Antiquité classique sur des espèces en régression
}

Suzanne Amigues

\section{To cite this version:}

Suzanne Amigues. Le témoignage de l'Antiquité classique sur des espèces en régression. Revue forestière française, 1991, 43 (1), pp.47-58. 10.4267/2042/26177 . hal-03425292

\section{HAL Id: hal-03425292 \\ https://hal.science/hal-03425292}

Submitted on 10 Nov 2021

HAL is a multi-disciplinary open access archive for the deposit and dissemination of scientific research documents, whether they are published or not. The documents may come from teaching and research institutions in France or abroad, or from public or private research centers.
L'archive ouverte pluridisciplinaire HAL, est destinée au dépôt et à la diffusion de documents scientifiques de niveau recherche, publiés ou non, émanant des établissements d'enseignement et de recherche français ou étrangers, des laboratoires publics ou privés. 


\title{
nature, hi \\ histoire,
}

\section{loisirs et forêt}

\section{LE TÉMOIGNAGE DE L'ANTIQUITÉ CLASSIQUE SUR DES ESPĖCES EN RÉGRESSION}

\author{
Suzanne AMIGUES
}

Grâce au plus ancien traité botanique connu, l'Historia plantarum (1) de Théophraste (c. 320-300 av. J.-C.), complété par des informations éparses dans la littérature gréco-latine moins spécialisée, nous sommes assez bien renseignés sur la distribution des essences forestières dans l'Est du bassin méditerranéen et au Proche-Orient quelques siècles avant et après le commencement de notre ère. Le but de cet article n'est pas d'esquisser le paysage forestier de la Grèce antique, ce que fit $P$. Buffault dans cette revue il y a plus d'un demi-siècle ${ }^{(2)}$, ni de développer mes précédentes remarques (Amigues, 1980) sur l'importance de la forêt et de ses produits dans la vie quotidienne des anciens Grecs, mais d'exposer le cas d'arbres ou arbustes aujourd'hui disparus de régions où leur présence est attestée de façon sûre par les textes anciens. II s'agit d'une part d'espèces réputées ouest-méditerranéennes dont l'aire s'étendait vers l'est bien au-delà de sa limite actuelle, et d'autre part des éléments qui composaient la mangrove du golfe Persique avant que celle-ci fût réduite à des peuplements purs d'Avicennia.

(1) Dénomination latine consacrèe par l'usage (sous la forme H.P. dans les références). Comme équivalent exact du titre grec Péri phytôn historia, j'ai adopté dans mon èdition en cours [Paris: Belles Lettres, 1988 (tome I, livres I-II) et 1989 (tome II, livres III-IV)] Recherches sur les plantes.

(2) Buffault (P.). - La Distribution des essences forestières en Grèce. - Revue des Eaux et Forēts, 69, 1931, pp. 217-229. 


\section{DES ESPĖCES JADIS OU NAGUĖRE PRÉSENTES EN MÉdITERRANÉE ORIENTALE}

\section{Le Chêne-liège (Quercus suber L.)}

Une allusion du poète tragique Eschyle aux "morceaux de liège (phelloi) qui, retenant le filet, sauvent des eaux profondes le réseau de lin" (Choéphores, v. 506-507) prouve que dès le $V^{e}$ siècle avant notre ère (la pièce date de 458) les pêcheurs grecs utilisaient le liège pour les flotteurs de leurs filets. Comment se le procuraient-ils?

Depuis le début du XIX siècle, aucun botaniste n'a pu constater la présence du Chêne-liège en Grèce. Fraas (1845, p. 254) dit de Quercus suber: "Devenu aujourd'hui très rare en Grèce (seulement çà et là en Arcadie), il est plus fréquent en Asie Mineure. Je ne l'ai moi-même jamais vu (...). Sibthorp et Bory Saint-Vincent ne l'ont pas trouvé non plus ". De même Boissier (1879, p. 1168): "Quercus suber: in Graecia raro (ex Fraas), in Albania (Griseb.). E ditione nondum vidi ". Halácsy (1904, p. 131) rapporte de manière hypothétique le Quercus suber de Fraas à Quercus crenata Lam. (= Quercus pseudo-suber Santi) dont l'existence à l'est de l'Albanie est elle-même mise en doute (Camus, 1936, p. 655) ou écartée (Schwarz dans Flora europaea, 1964. p. 63). "En tout cas actuellement l'absence de Quercus suber dans les Balkans et à plus forte raison en Anatolie est certaine pour tous les botanistes et forestiers " (P. Quézel, communication personnelle du 26-10-1983) ; cf. aussi la distribution de l'espèce dans Flora europaea, loc. cit.: "S. Europe, from N.-W. Jugoslavia westwards".

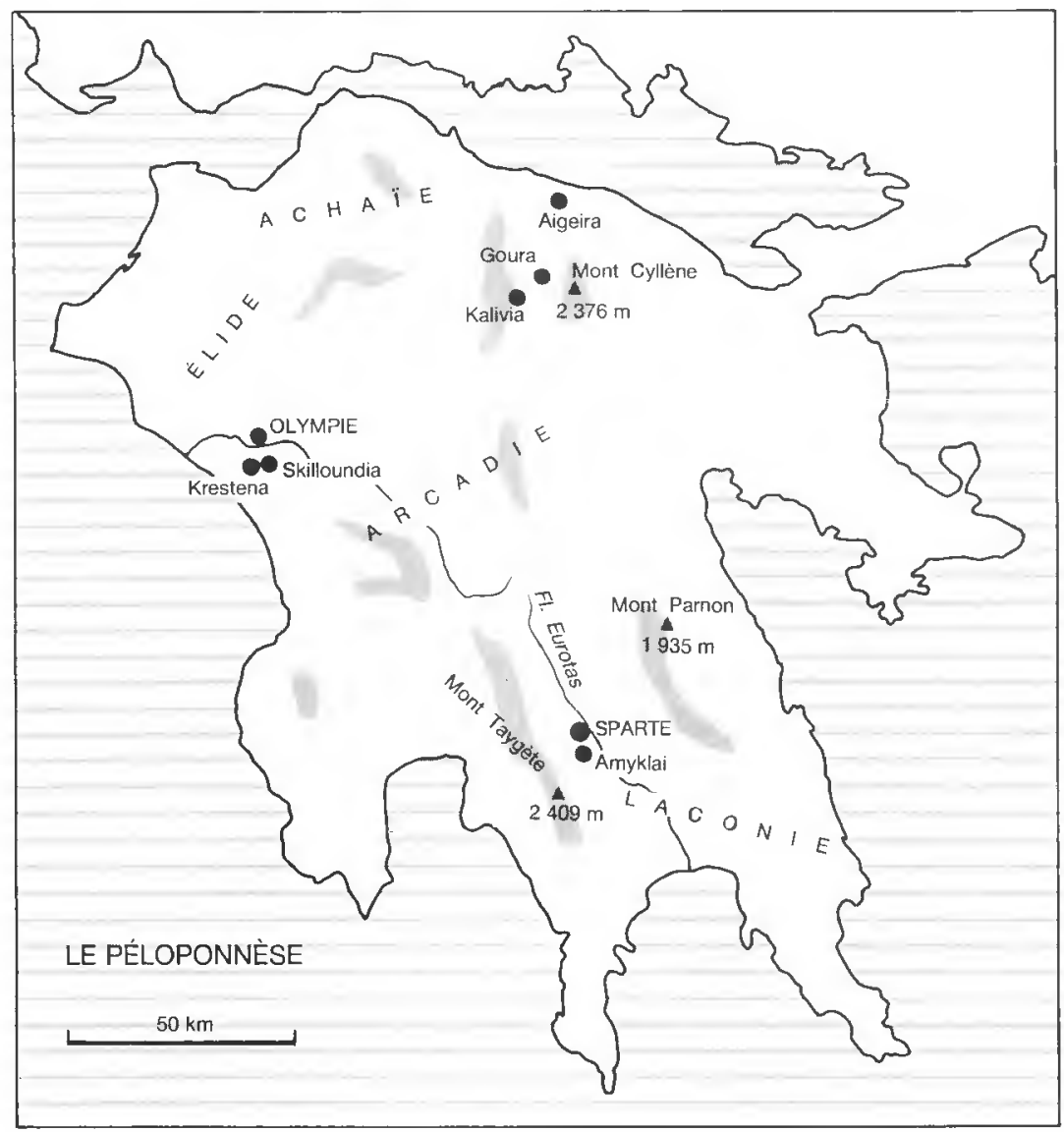


Cependant, d'après les sources antiques, Quercus suber se rencontrait encore dans le Péloponnèse au $\|^{e}$ siècle de notre ère. Le plus ancien témoignage, sinon le plus clair, est celui de Théophraste qui décrit séparément "l'arbre que les Arcadiens appellent Chêne-liège (phellodrys) " (H.P. III, 16, 3) et une espèce qu'il nomme littéralement "le liège " (phellos) et tient pour propre à l'Étrurie (H.P. III, 17, 1). La seconde est incontestablement Quercus suber (malgré la mention de feuilles caduques due à un renseignement inexact ou plutôt à une confusion avec Quercus pseudo-suber), car l'auteur lui attribue "une écorce fort épaisse" au sujet de laquelle il précise: "On lève l'écorce à la périphérie du tronc et l'on prétend qu'il faut la détacher toute, sinon l'arbre dégénère. Elle se reconstitue totalement à peu près en trois ans". En revanche, la description assez embarrassée du phelladrys arcadien ne conduit pas à une identification certaine. On en retient qu'il s'agit d'une espèce sempervirente, proche du Chêne kermès (dans sa forme naguère dénommée Quercus calliprinos Webb) pour les feuilles, les glands, les caractères du bois, la couleur rouge-brun du tronc écorcé. "Aussi l'utilise-t-on, ajoute Théophraste, là où ne pousse pas le Chêne kermès, pour le charronnage et les travaux de ce genre, comme cela se fait en Laconie et en Élide. Les Doriens, eux, donnent également à cet arbre le nom de Chêne vert". Aucune mention n'est faite de l'écorce, ce qui suppose que si le phellodrys de Théophraste correspond à Quercus suber, son liège n'était pas l'objet à cette époque d'une exploitation organisée. Nous serons mieux renseignés par le voyageur-archéologue Pausanias qui a parcouru l'Arcadie au $I^{e}$ siècle après J.-C. et noté (Description de la Grèce, VIII, 12, 1) avec sa précision coutumière les noms d'arbres les plus remarquables de la région: "Dans les chênaies d'Arcadie, les chênes appartiennent à différentes espèces: on appelle certains d'entre eux "chênes à larges feuilles" (= Quercus frainetto), les autres "chênes à glands comestibles" (= Quercus aegilops). Ceux de la troisième espèce présentent une écorce poreuse et naturellement si légère qu'on en fait en mer des balises pour les ancres et pour les filets. C'est l'écorce de ce chêne qu'entre autres loniens le poète élégiaque Hermèsianax nomme liège (phellos) ". L'utilisation de l'écorce n'eût pas été possible si, comme le suggérait Halácsy, le "Chêne-liège" d'Arcadie était Quercus pseudo-suber, qui produit un liège trop mince et trop fragile. On est donc tenté d'admettre, sur la foi de Pausanias, l'identité du phellodrys antique et de Quercus suber.

II reste à se demander si les régions du Péloponnèse où les textes anciens situent cette espèce (l'Arcadie, la Laconie ou pays de Sparte, l'Élide qui s'étend aux alentours d'Olympie) offrent des conditions édaphiques et phytosociologiques permettant de penser que Quercus suber a pu effectivement s'y trouver. Cette question se pose en particulier pour les lieux désignés autrefois par des noms dérivés de phellos, donc susceptibles de correspondre à des stations isolées de Chênes-liège dans les provinces limitrophes de l'Arcadie: on sait en effet que l'exception laisse dans la toponymie plus de traces que la norme. En Laconie, Pausanias signale (III, 20,3) un cours d'eau nommé Phellia qui descend du Taygète et se jette dans l'Eurotas en amont d'Amyklai, à peu de distance au sud de Sparte. Précisément ce piémont oriental du Taygète est schisteux et porte une végétation calcifuge à Erica arborea et Arbutus unedo (Barbero-Quézel, 1976, p. 24 et tableau 8) que nous voyons associés à Quercus suber dans les Albères et les Maures. D'autre part, à proximité d'Olympie se trouvait, selon le géographe Strabon (VIII, 3, 14), un lieu-dit Phellôn, soit probablement "la suberaie", la formation du nom étant parallèle à celle de daphnôn "le bois de lauriers", élaôn "l'oliveraie ", etc. C'est dans les collines voisines de Krestena-Skilloundia, où l'acidité du sol se manifeste par l'abondance de la Fougère aigle, qu'on s'accorde à placer le Phellôn de Strabon. Il y avait enfin au nord de l'Arcadie, dans l'arrièrepays d'Aigeira en Achaïe, une petite ville du nom de Phelloè que Pausanias décrit (VII, 26, 10) entourée de vignes et de bois de chênes; certes, il n'est pas dit que ceux-ci étaient des Chênes-liège, mais le site présumé de Phelloè confine à la région de Goura-Kalivia (à l'ouest du Cyllène) où le Châtaignier et la Bruyère en arbre indiquent en maints endroits l'absence de calcaire actif (Barbero-Quézel, tableau 9).

En résumé, les textes et la roponymie permettent d'imaginer de la manière suivante la distribution de Quercus suber dans la Grèce antique: en Arcadie le phellodrys constituait un élément 


\section{Suzanne AMIGUES}

appréciable des chênaies et son liège fut localement exploité au moins vers le début de notre ère ; sur le pourtour de cette province, il formait çà et là de petits peuplements d'où on ne tirait pas de produit spécifique et il n'y était même pas toujours clairement distingué, dans les faits et dans les mots, du Chêne vert dont l'aire actuelle comprend précisément l'arrière-pays d'Olympie et une partie des contreforts du Taygète (Barbero-Quézel, p. 18 et tableau 6). Notons à ce sujet une coïncidence remarquable entre les données antiques et ces observations de Barbero-Quézel (p. 28) sur le Cytiso-Castanetum de Laconie (Taygète et Parnon): "Diverses espèces des Quercetalia ilicis apparaissent également et Erica arborea est fréquente. Ce groupement n'est pas sans rappeler l'ambiance de certaines forêts de Quercus suber en Méditerranée occidentale". Dans ces conditions, il semble raisonnable de se ranger à l'opinion de Camus (1936, p. 494, n. 3): "Le Quercus suber était autrefois en Grèce: les Grecs importaient cependant d'italie une grande partie du liège qu'ils employaient".

\section{Le Palmier nain (Chamaerops humilis L.)}

Traitant des palmiers (H.P. II, 6), Théophraste fait une allusion à Chamaerops humilis à propos des fruits, qui sont chez certaines espèces " aussi petits que des pois chiches" (§6), avant de préciser ( $(11)$ : "Ceux des palmiers qu'on dit nains (chamaerripheis) constituent un autre genre, en quelque sorte homonyme [s.e. des palmiers à stipe]. Quand on leur a coupé le cœur, ils continuent à vivre; coupés à ras des racines, ils forment des rejets. Ils se distinguent à la fois par leurs fruits et par leurs feuilles: ils ont la feuille plane et souple, ce qui permet de l'utiliser pour tresser couffins et paniers. Communs en Crète, ils le sont encore plus en Sicile". Pline (XIII, 39), à qui Linné a emprunté le nom générique du Palmier nain, se borne par ailleurs à résumer Théophraste: "Les Grecs nomment chamaerops un palmier bas à feuilles plus larges et plus souples très utilisées en sparterie, qui abonde en Crète et plus encore en Sicile".

Il est donc ainsi établi par un témoignage en fait unique mais formel que Chamaerops humilis prospérait en Crète au IV siècle avant notre ère. Hormis Sfikas (1981, p. 194) pour qui "le Palmier nain se trouve à l'état spontané dans plusieurs stations de Crète", les botanistes modernes et contemporains s'accordent sur son absence actuelle de tout le monde grec. Ainsi notamment Boissier (1884, p. 47), Gandoger (1916, p. 177), Gennadios (1959, p. 956), Polunin Huxley (1967, p. 264), J. do A. Franco dans Flora europaea (1980, p. 267), P. Quézel (communication personnelle du 26-10-1983). Déjà Tournefort (1717, p. 56) n'avait pas retrouvé en Crète les palmiers signalés par Théophraste.

Comme l'extinction de Quercus suber en Grèce, celle de Chamaerops humilis en Crète s'explique à la fois par une exploitation abusive et par la fragilité naturelle d'une espèce sur les marges de son aire.

Les feuilles souples et résistantes du Palmier nain constituaient un matériau recherché : l'économie antique exigeait la fabrication d'innombrables articles de sparterie pour le transport des olives, des raisins et autres fruits, du poisson, etc...; il est probable d'autre part, malgré le silence de nos sources, que les fibres effilochées des feuilles servaient jadis comme encore aujourd'hui à faire des cordages, et les Crétois étaient par excellence des marins. Pline note aussi $(X I I I, 28)$ que "dans la plupart des régions, la feuille sert à protéger les murs des averses à la place d'un crépi". Quant à l'usage alimentaire du "cœur de palmier", plusieurs fois mentionné pour le dattier (Xénophon, Anabase, II, 3, 16 ; Arrien, Inde, XXIX, 1 ; etc...), it paraît se déduire pour le Palmier nain de la remarque de Théophraste: "quand on leur a coupé le cœur, ils continuent à vivre". Les Siciliens en tout cas ne dédaignaient pas cette ressource naturelle, d'après le commentaire de Servius à Virgile, Énéide, III, vers 705: "Sélinonte est une ville (...) où abondent des palmiers dont les gens se nourrissent". Encore au siècle dernier les parties tendres des bourgeons, pétioles et spadices de Chamaerops humilis étaient couramment consommées "en Sicile, en Algérie, et dans tous les pays où croît cette plante" (Lenormant, 1856, p. 151). Il est même arrivé, en cas de disette extrême, que des hommes affamés déterrent les 
racines pour s'en nourrir (Ciceron, $2^{e}$ Action contre Verrès, V, 87). Ajoutons à ces usages, déjà suffisants pour mettre l'espèce en péril, la possibilité d'un défrichement précoce de zones littorales sablonneuses favorables à Chamaerops humilis, par exemple dans la région d'lérapétra aujourd'hui couverte de cultures maraîchères. En effet, les mutilations auxquelles Théophraste affirme que ce palmier survit dénoncent peut-être autant qu'une exploitation maladroite un effort délibéré de destruction.

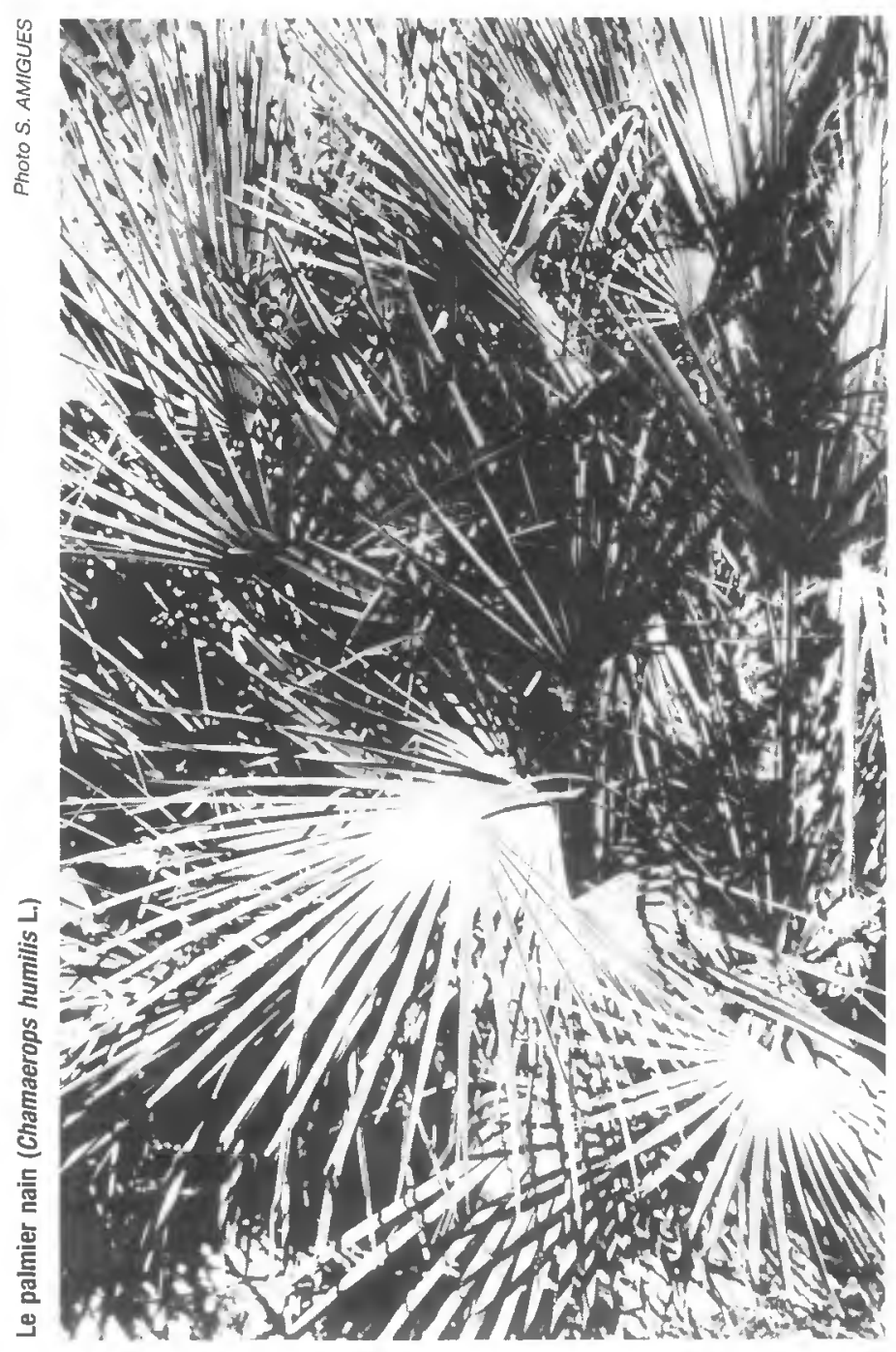

Sans minimiser le rôle déterminant de l'homme dans l'extinction de Chamaerops humilis en Crète, on peut penser que ce dernier, malgré sa vitalité, n'était pas là aussi solidement implanté que dans le Sud du bassin méditerranéen occidental, son domaine d'élection. II a de même disparu à l'état spontané de l'extrémité septentrionale de son aire, à savoir la région niçoise oủ "Il existait pourtant au milieu du XIX siècle sur les rochers entre Monaco et Saint-Alban" (Harant-Jarry, 1963, p. 199 ; voir aussi Daveau, 1899, p. 4 ; Combes, 1903, p. 236). 


\section{Suzanne AMIGUES}

\section{Le Thuya de Barbarie (Tetraclinis articulata (Vahl) Masters)}

La régression du Thuya de Barbarie (Tetraclinis articulata (Vahl) Masters = Callitris articulata (Vahl) Link, Callitris quadrivalvis Vent.) est analogue à celle des deux espèces précédemment étudiées, mais encore plus frappante car cette essence, aujourd'hui considérée comme un élément ibéro-maurétanien (Quézel-Santa, 1962, p. 34), constituait dans l'Antiquité des peuplements étendus en Cyrénaïque et se trouvait même dans l'oasis de Siwa, en plein désert libyque.

Le premier, Théophraste (H.P. V, 3, 7) donne de cet arbre une description assez précise et vante les qualités de son bois, recherché pour les charpentes et l'ébénisterie: "Le thyon, autrement dit le thuya, croit aux abords du sanctuaire ammonien [i.e. du temple de Zeus - Ammon à Siwa] et sur le territoire de Cyrène; il a l'aspect d'un cyprès pour les rameaux, les feuilles, le tronc et le fruit, mais c'est plutôt comme un cyprès sauvage [i.e. Cupressus sempervirens L. $f$, horizontalis (Miller) Voss]; il abondait en particulier sur le site actuel de la ville et on se rappelle encore que dans l'ancien temps certaines toitures en étaient faites. Le bois en général est imputrescible mais la partie la plus madrée est la racine, dont on fait les ouvrages les plus soignés". L'exploitation du thuya libyen a donc commencé dès la fondation de Cyrène par des colons grecs de Théra, aux environs de 631 avant J.-C. (Chamoux, 1952, p. 124). Son premier emploi, comme matériau de construction, était purement utilitaire et sans doute local ; mais la mention d'“ ouvrages soignés " faits avec le bois madré des racines annonce déjà la vogue extraordinaire que devaient connaître, surtout à l'époque romaine, les objets de luxe en thuya.

Après son annexion par Alexandre, la Cyrénaïque passa sous l'autorité des souverains lagides et ses bois les plus précieux prirent le chemin d'Alexandrie. Ainsi la cabine du bateau somptueux construit pour Ptolémée Philopator, roi d’Égypte de 221 à 203, avait, nous dit Athénée (Les Deipnosophistes, 205b) des portes en thuya. Quelque cent-cinquante ans plus tard, la Cyrénaïque avait probablement cessé de produire un bois de qualité puisque, selon Lucain (La guerre civile, $X$, vers 144-146), il y avait dans le palais où Cléopâtre reçut César en 48 avant J.-C. des tables rondes à pieds d'ivoire " en bois de l'Atlas ".

Pour le $1^{\text {er }}$ siècle de notre ère, notre principale source de renseignements est la longue notice (XIII, vers 91-102) que Pline consacre au "citre" (en prenant soin de signaler (\$100) la synonymie des noms grecs thyon/thya et du latin citrus), à son bois et particulièrement à " cette

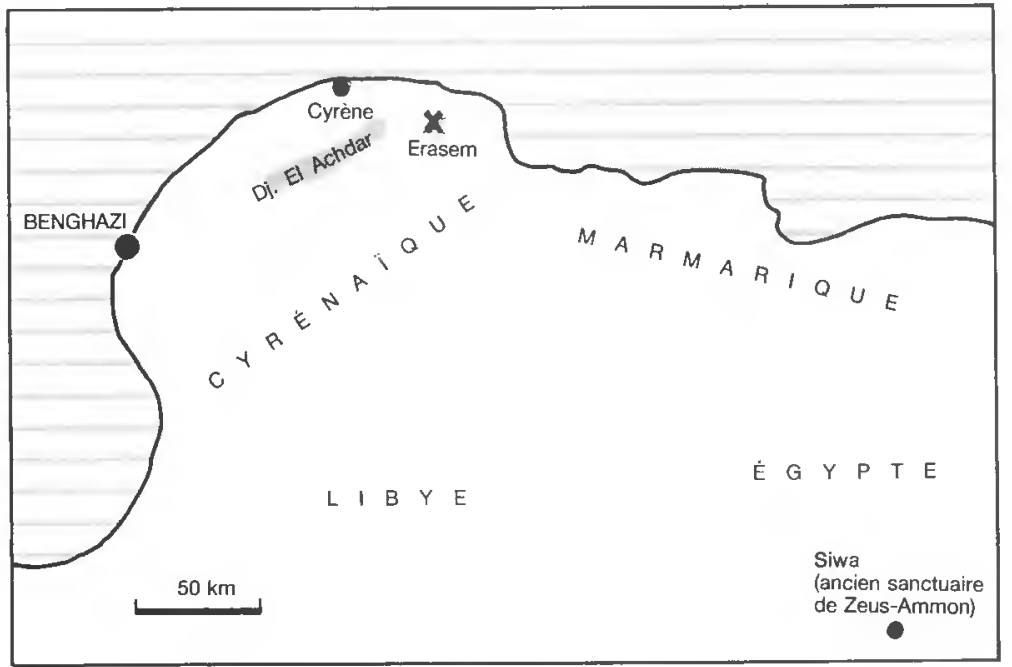




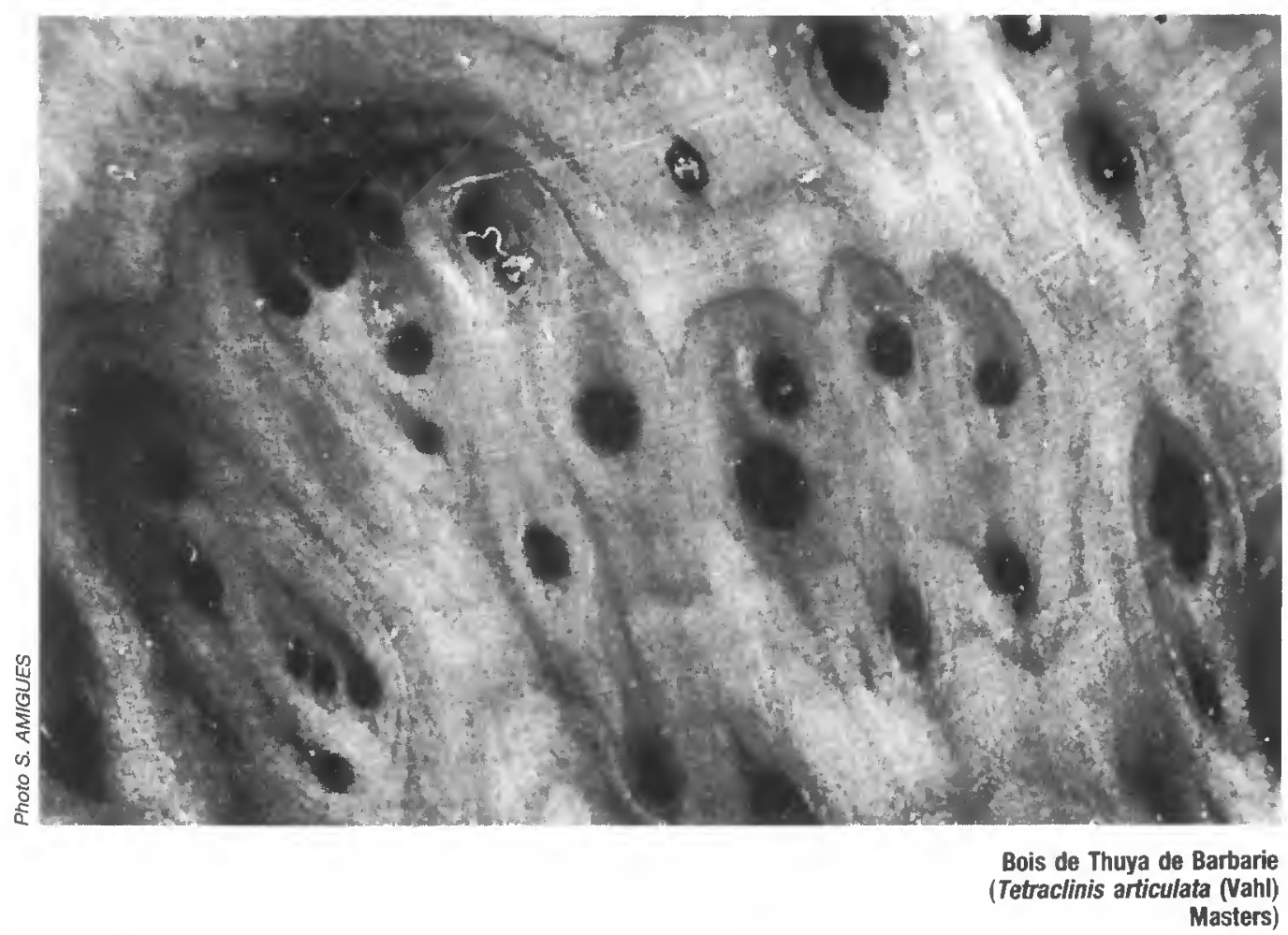

folie des tables de citre que les femmes rétorquent aux hommes quand ils leur reprochent leurs perles " (\$91). Les plateaux de ces tables, dont certaines dépassaient de beaucoup le diamètre de simples guéridons (§93-94), étaient taillés dans les loupes développées en terre ou au ras du sol sur la souche de l'arbre. Les amateurs recherchaient surtout (§96-97) les madrures " en forme de cheveux crépus ou de petits chignons", mais tenaient aussi en grande estime celles qui rappelaient "les ocelles des queues de paon"; la couleur la plus appréciée était la teinte chaude du vin miellé, et naturellement la valeur d'une section de loupe sans défaut augmentait en proportion de sa taille. Les plus belles pièces coûtaient de véritables fortunes: les prix que Pline a notés (\$92) vont de 500000 sesterces, soit environ 100000 francs or, pour la table de Cicéron, à 1300000 sesterces, "la valeur d'un grand domaine", pour un exemplaire appartenant à la famille des Céthégus.

II est possible de situer approximativement dans le temps et plus précisément dans l'espace l'exploitation massive de Tetraclinis articulata consécutive à cette mode. À sa traduction de H.P. V, 3, 7, Pline ajoute cette remarque (fin $\S 102$ ): "Théophraste ne dit mot des tables de citre, et l'on n'en mentionne nulle part avant celle de Cicéron; ce qui prouve qu'elles sont récentes". Comme Cicéron mourut en 43 avant J.-C. et que la médiocrité de sa fortune ne dut lui permettre qu'assez tard une telle dépense, l'engouement des Romains pour les citreae mensae remonte environ au milieu du $1^{\text {er }}$ siècle avant notre ère. À cette époque, nous l'avons vu, des tables de thuya maurétanien meublaient déjà les palais d'Alexandrie, mais ce n'était encore qu'un luxe princier. Dès lors les Romains allaient mettre au pillage les forêts de l'actuel Maghreb. Si Lucain (IX, vers 426-430) le déplore avec une grandiloquence qui peut rendre son témoignage suspect d'exagération, de son côté, Pline l'indique expressément (§91): “ $\dot{A}$ l'Atlas confine la Maurétanie, où abondent les citres, et d'où vient cette folie des tables de citre, 
etc... ". Pline ayant écrit son Histoire naturelle entre 50 et 79 de notre ère, on voit avec quelle rapidité Tetraclinis articulata régressait dans le Maghreb même, d'après ce détail précis (§ 93$)$ : "C'est le mont Ancorarius, dans la Maurétanie intérieure, qui produisait le citre le plus estimé ; il est déjà épuisé ". Si l'Ancorarius est l'actuel Ouarsenis (Gsell, 1913, p. 140) où des peuplements de thuya existent encore (Despois, 1949, p. 89), il faut entendre qu'on n'y trouvait plus, à l'époque de Pline, de vieux sujets porteurs de loupes énormes, mais les arbres jeunes n'y manquaient pas pour autant.

Qu'advint-il de Tetraclinis articulata en Cyrénaïque après des siècles d'exploitation intensive sous la domination lagide? J.-R. Pacho $(1827$, p. 83) remarquait en gravissant pendant I'hiver 1824-1825 le versant oriental des montagnes cyrénéennes des "forêts de genévriers rembrunis, de verdoyants thuyas et de pâles oliviers". II devait revoir plus loin (cf. ibid., p. 87), dans la région de l'antique Irasa qu'il identifie avec Erasem, les mêmes essences formant une épaisse forêt dont l'étendue correspondait à deux heures de marche. Cependant, moins d'un siècle plus tard, Durand et Barratte (1910, p. CV) incluent Callitris quadrivalvis Rich. (= Tetraclinis articulata) dans leur "liste des plantes qui n'ont pas été retrouvées depuis la publication, en 1824, du Florae Libycae specimen de Viviani". Les données les plus récentes sont elles-mêmes contradictoires : cette espèce est absente de la Checklist de Keith (1965), mais présente pour la Libye dans Med-Checklist 1 (1984, p. 28), et un ouvrage de vulgarisation largement diffusé (Polunin Huxley, 1967, p. 76) indique de "petits peuplements isolés (...) en Cyrénaïque". Nous admettrons sans grand risque d'erreur que Tetraclinis articulata y est aujourd'hui très raréfié, sinon complètement éteint.

Son évolution dans l'Est du bassin méditerranéen est donc parallèle à celle de Quercus suber et de Chamaerops humilis, sauf que sa régression présente plus d'ampleur s'il est vrai que la limite orientale de son aire s'est déplacée de Siwa au IV siècle avant J.-C. à Malte et à l'est du Maghreb de nos jours.

\section{LA MANGROVE DU GOLFE PERSIQUE VUE PAR LES COMPAGNONS D'ALEXANDRE}

Quand Alexandre, après avoir franchi l'Indus et le Jhelum, fut contraint par ses troupes à rebrousser chemin, il descendit le cours de I'Indus et arriva en juillet 325 avant J.-C. à Pattala (Hyderabad?) où il passa la fin de l'été à préparer sa flotte au long parcours entre les bouches de l'Indus et celles de l'Euphrate. Les naturalistes de l'expédition, en particulier Androsthène de Thasos qui semble avoir été le principal informateur de Théophraste sur ce sujet, découvrirent alors les mangroves du delta de l'Indus. Faute de pouvoir en nommer les essences, ils les ont décrites à l'aide de comparaisons bien choisies qui nous permettent de les identifier. II s'agit en effet de mangliers (Rhizophora mucronata Lam.) dans H.P.IV, 7, 4: " $\dot{A}$ marée montante, alors que tout le reste disparaît, on voit émerger les rameaux des arbres les plus élevés, auxquels on attache les amarres, qui sont ensuite, au moment du reflux, attachées aux racines; ces arbres ont la feuille du laurier, une fleur dont la teinte et l'odeur ressemblent à celles des violiers, un fruit de la grosseur d'une olive, lui aussi délicieusement parfumé; ils ne perdent pas leurs feuilles; la fleur et le fruit se forment quand vient l'automne et tombent au printemps ". Dans la mer également, les observateurs antiques ont remarqué (H.P. IV, 7, 5) des arbres à feuilles persistantes et fruits semblables à ceux du Lupin; on y reconnaît à la suite de Bretzl (1903, p. 60-62) Aegiceras corniculatum (L.) Blanco (= Aegiceras majus Gaertn.), dont les fruits en forme de corne rappellent effectivement les légumes d'une Papilionacée. Aujourd'hui encore Rhizophora mucronata et Aegiceras corniculatum entrent dans la composition des mangroves de l'Indus (Stewart, 1972, pp. 501 et 532) qui, autant qu'on peut en juger d'après ces deux espèces, est restée assez stable depuis l'Antiquité. 
Dans le golfe Persique, les formations analogues se sont au contraire extrêmement appauvries au cours du même laps de temps. Quand la flotte d'Alexandre eut passé le détroit d'Ormuz, elle stationna dans les parages de Qeshm à la fin de l'année 325, pendant que son commandant faisait sa jonction avec l'armée de terre venue du Baluchistan à travers le Makran. Les naturalistes grecs purent y observer à loisir l'Avicennia marina (Forsk.) Vierh. (- Avicennia officinalis L.) parfaitement reconnaissable dans H.P.IV, 7, 5: "En Perse, dans la province de Carmanie où la marée se fait sentir, il existe des arbres d'une bonne taille, qui ont le port et les feuilles de l'Arbousier d'Orient (= Arbutus andrachne L.) et produisent en abondance des fruits semblables extérieurement à nos amandes, mais dont l'intérieur a des replis, comme s'il était comprimé de toutes parts".

L'Avicennia existe toujours à Qeshm et sur la côte voisine, mais en peuplements purs (Kunkel, 1977, p. 74 ; Rechinger, 1978 , p. 2), alors que les compagnons d'Alexandre l'ont vu mêlé à Rhizophora mucronata et à Aegiceras corniculatum déjà observés aux bouches de l'Indus. C'est en effet au Rhizophora qu'appartiennent les racines-échasses bien décrites dans H.P. IV, 7 , 5: "Ces arbres ont le milieu rongé en dessous par la mer et ils se dressent sur leurs racines comme un poulpe. Au moment du reflux, il est possible de s'en rendre compte". On retrouve également ( $\$ 6$ ) Aegiceras corniculatum avec son "fruit pareil à celui du Lupin ", mais un détail nouveau, la "fleur agréablement odorante", vient confirmer l'identification de cette espèce naguère nommée Aegiceras fragrans König.

\section{Avicennia marina (Forsk.) Vierh.}

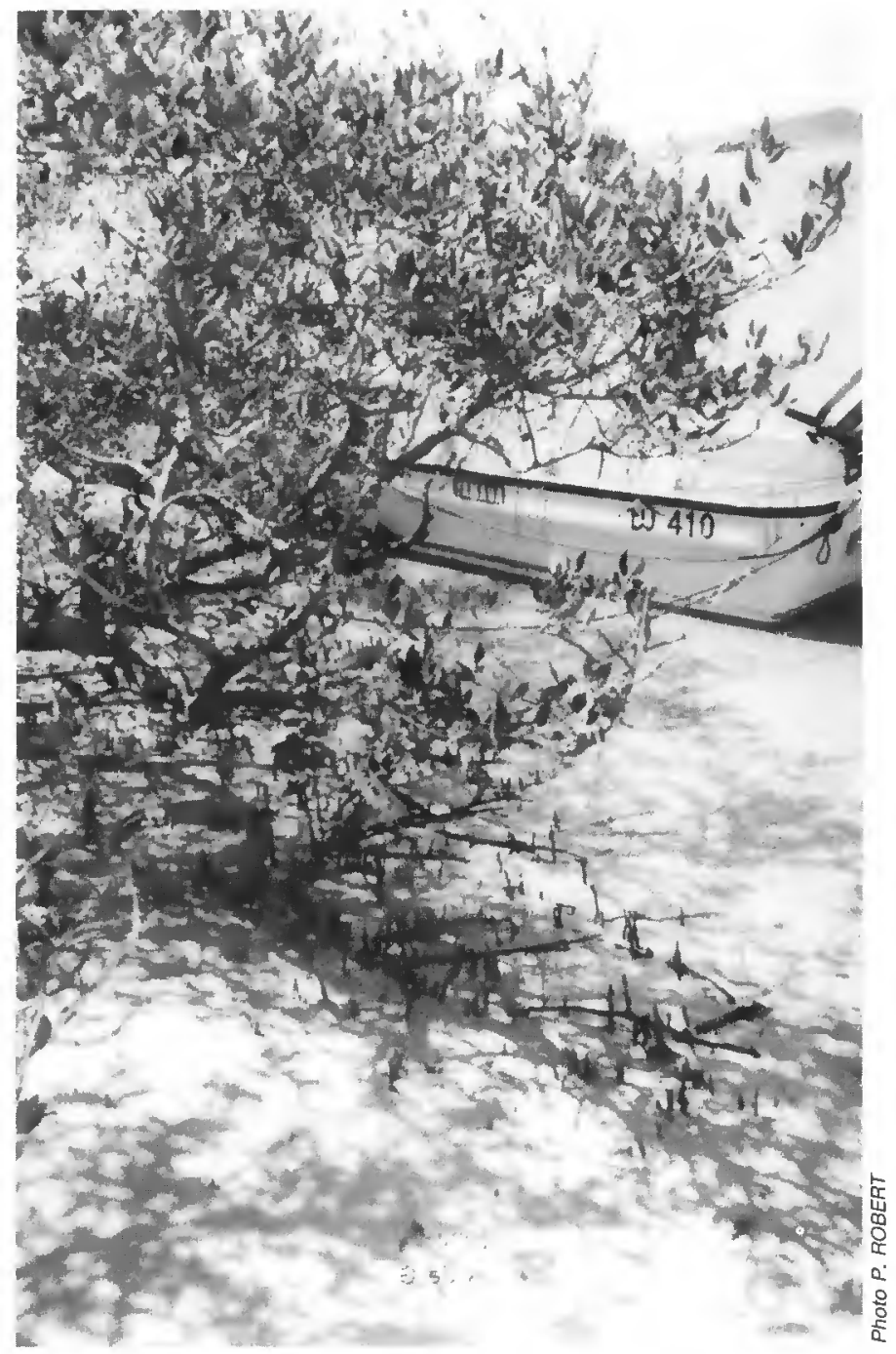

De retour à Babylone, Alexandre torma le projet d'établir des colonies sur la côte occidentale du golfe Persique; en 324, il confia une mission de reconnaissance à Androsthène, que Théophraste cite nommèment (De causis plantarum, II, 5, 5) comme la source de ses informations sur Tylos, l'actuelle Bahrein. Androsthène revit là encore, et en grande abondance, Aegiceras 


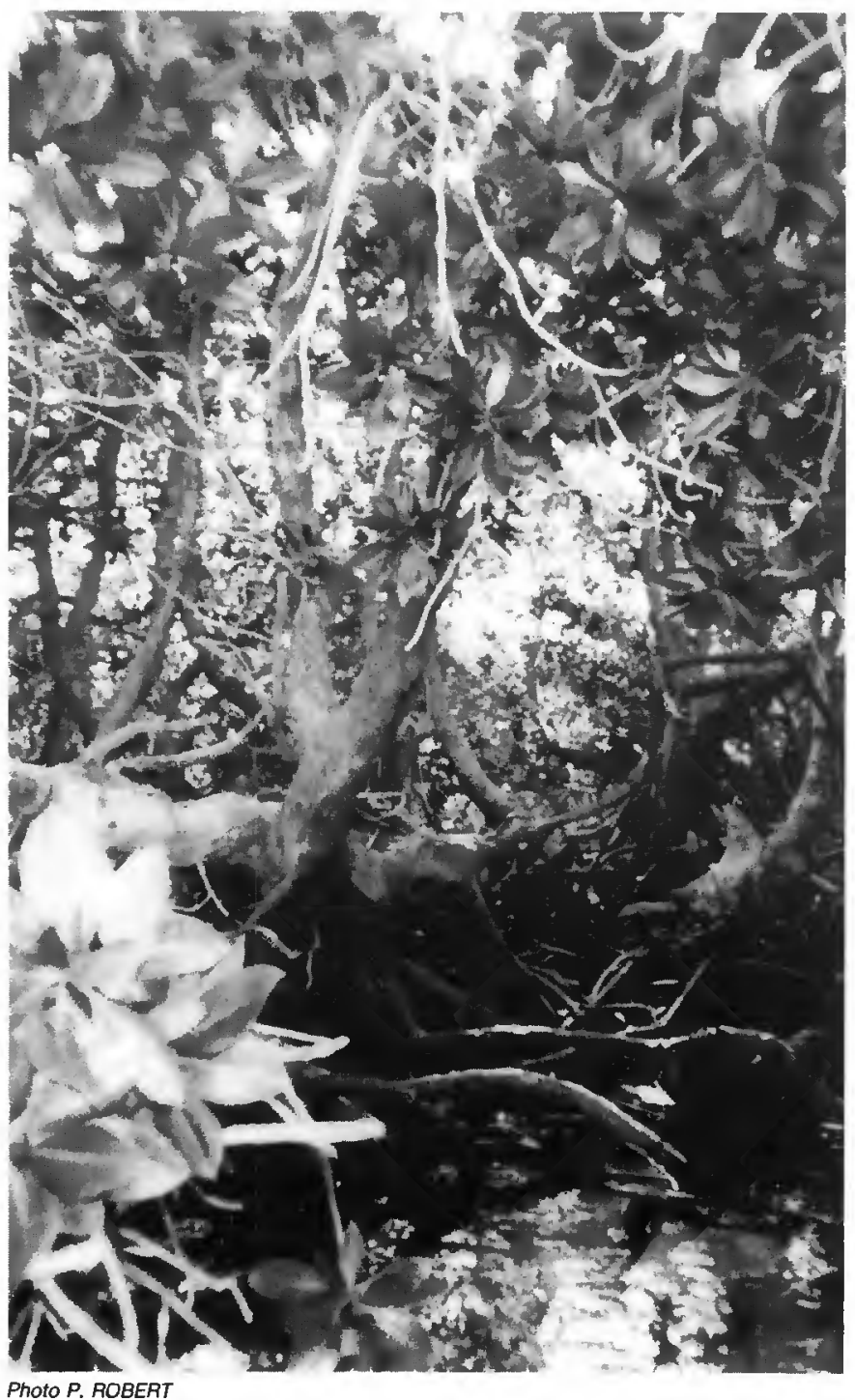

Tronc rongé et racines-échasses de Rhizophoracée.

corniculatum (H.P. IV, 7, 7): "Dans l'île de Tylos, il y a sur la côte orientale, au moment oú la mer se retire, une telle quantité d'arbres que l'ile se trouve fortifiee; tous ces arbres ont la taille d'un figuier, une fleur extraordinairement parfumée, un fruit non comestible, semblable d'aspect à celui du Lupin ". À Tylos ègalement, ajoute Théophraste (H.P. IV, 7, 8), croît "encore un autre arbre dont la fleur a de nombreux pétales, comme la rose; cette fleur, dit-on. reste close pendant la nuit, elle s'ouvre au lever du soleil, elle est à midi complètement épanouie, puis l'après-midi elle se referme peu à peu pour, de nouveau, rester close pendant la nuit; et même les indigènes disent qu'elle dort ". La comparaison avec la rose rappelie une remarque isolée dans un chapitre de morphologie (H.P. I, 13, 1) où

l'auteur constate que la plupart des arbres ont des fleurs blanches, sauf le Grenadier, certains Amandiers, le Sapin, "sans parler de celles qui, dit-on, dans la mer Extérieure [entendons une mer autre que la Méditerranée] ont la couleur des roses". S'il est permis d'attribuer aux fleurs d'une seule et même espèce d'arbre poussant au bord d'une mer exotique les caractères cidessus énumérés : pétales nombreux, couleur rose, port variable selon l'éclairement, l'espèce en question est très probablement Bruguiera gymnorrhiza Lam. Celle-ci coexiste avec toutes les précédentes en particulier dans le delta de l'Indus (Stewart, 1972, p. 501 ; Chapman, 1976, p. 105).

Une dernière indication sur la composition floristique de la mangrove de Tylos est fournie par H.P. V, 4, 7: " I y a, paraît-ll, un arbre dans lequel les gens taillent leurs cannes, qui sont fort belles avec une bigarrure semblable à celle de la peau du tigre; ce bois est extrêmement lourd, mais qu'on le lance contre une surface un peu dure, il se brise comme la poterie". Pline (XVI, 
§ 221) ajoute à Théophraste deux précisions intéressantes : il s'agit en fait d'un arbrisseau ; son bois est "fragile comme du verre" (uitri modo fragilem). Ces caractères si originaux se retrouvent intégralement dans Excaecaria agallocha L., un petit arbre des tidal forests asiatiques et océaniennes, qui possède un bois "d'un brun rougeâtre, jaspé de gris ou de noir, dur, pesant, fragile " (Baillon, 1874, p. 175), "dur et fragile comme du verre" - ce sont les propres termes de Pline - (Beauverie, 1910, p. 314).

En résumé, les mangroves du golfe Persique comprenaient en 325-324 au moins cinq espèces: Aegiceras corniculatum, Avicennia marina, Bruguiera gymnorrhiza, Excaecaria agallocha, Rhizophora mucronata. Toutes ont aujourd'hui disparu, sauf l'Avicennia, présent à Bahrein (Dickson, 1955, p. 25) comme sur la côte orientale.

La gravité de cet appauvrissement est due pour une part à l'influence humaine: dès l'époque de Théophraste, les habitants de Tylos faisaient leurs cannes en bois d'agalloche, leurs bateaux en palétuvier (H.P. V, 4, 7: "Dans l'île de Tylos qui avoisine l'Arabie, il est, dit-on, un bois utilisé à la construction des bateaux, qui est presque incorruptible en mer: il se conserve plus de deux cents ans au fond de l'eau; à l'air libre, il est durable mais pourrit plus vite") ; les médecins utilisaient le suc de Rhizophora pour préparer une drogue hémostatique (H.P. IV, 7, 2). Si l'on tient compte aussi des besoins domestiques en matériau de construction, combustible, menus objets d'usage quotidien, ainsi que des ravages causès par la voracité des chèvres et des dromadaires (Ibn-Hassân ap. Ibn-el-Baytar, in Leclerc, 1877-1883, nº 1981 ; Baillon, 1892, p. 95 ; Chapman, 1976, p. 87), on imagine à quelle épreuve la mangrove fut et reste soumise dans les régions désertiques du Proche-Orient.

Les conditions climatiques jouent d'autre part un rôle important que souligne Chapman (1976, p. 104) : "Certaines régions, par exemple le Sind et le golfe Persique, sont extrêmement arides et le développement de la mangrove est toujours médiocre dans de telles conditions". Seul l'Avicennia s'accommode assez bien de cette aridité et remonte en mer Rouge jusqu'à l'extrémité méridionale de la péninsule du Sinaï. Les formations comprenant entre autres les cinq espèces décrites par Théophraste se rencontrent aujourd'hui dans I'Inde occidentale (entre 60 et $75^{\circ}$ de longitude Est), sous un climat tropical plus humide (Tomlinson, 1986, tableau 3.2).

Ainsi donc dans le golfe Persique comme en Méditerranée, ce sont les espèces les moins solidement établies à l'extrémité de leur aire qui ont le plus souffert de l'influence humaine. Aux spécialistes de dire s'il serait actuellement possible de réintroduire certaines d'entre elles dans des régions qu'elles occupaient il y a deux millénaires. Les observations minutieuses de Théophraste et de ses informateurs nous permettent d'évaluer l'ampleur de leur régression. La solution du problème, s'il en est une, appartient à notre temps.

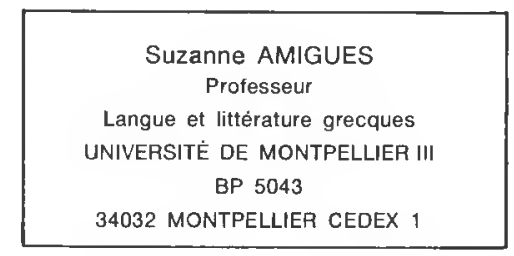




\section{BIBLIOGRAPHIE}

AMIGUES (S.). - Quelques aspects de la forêt dans la littèrature grecque antique. - Revue forestière française, vol. XXXIII, $\mathrm{n}^{\circ} 2,1980$, pp. 211-223,

BAILLON (H.). - Histoire des plantes. - Paris, tome V, 1874 ; tome XI, 1892.

BARBERO (M.), QUÉZEL (P.). - Les Groupements forestiers de Grèce centroméridionale. - Ecologia Mediterranea, $\mathrm{n}^{\circ} 2,1976, \mathrm{pp} .3-86$.

BEAUVERIE (J.). - Les Bois industriels. - Paris, 1910.

BOISSIER (P.E.). - Flora orientalis, - Basileae, Genevae et Lugduni. - Tome IV, $1879 ;$ tome V, 1884.

BRETZL (H.). - Botanische Forschungen des Alexanderzuges. - Leipzig, 1903.

CAMUS (A.). - Les Chênes. - Tome I. - Paris, 1936.

CHAMOUX (F.). - Cyrène sous la monarchie des Battiades. - Paris, 1952.

CHAPMAN (V.J.). - Mangrove Vegetation. - Vaduz, 1976.

COMBES (P.). - Le Palmier nain. - Cosmos, $n^{\circ}$ 969, 1903, pp. 236-237.

DAVEAU (J.). - Le Palmier nain et le Caroubier en Portugal. - Montpellier, 1899.

DESPOIS (J.). - L'Afrique du Nord. - Paris, 1949.

DICKSON (V.). - The Wild Flowers of Kuwait and Bahrain. - London, 1955.

DURAND (E.), BARRATTE (G.). - Extrait du Florae Libycae Prodromus ou Catalogue raisonné des Plantes de Tripolitaine. - Genève, 1910.

FLORA europaea / T.G. Tutin, V.H. Heywood et coll. - Cambridge, tome I, 1964 ; tome V, 1980.

FRAAS (C.). - Synopsis plantarum florae classicae. - München, 1845.

GANDOGER (M.). - Flora cretica. - Paris, 1916.

GENNADIOS (P.). - Lexicon phytologicon (en grec). - $2^{\mathrm{e}}$ edition. - Athenai, 1959.

GSELL (S.). - Histoire ancienne de l'Afrique du Nord. - Paris, tome I, 1913.

HALACSY (E. de). - Conspectus Florae Graecae. - Lipsiae. - Tome III, 1904.

HARANT (H.), JARRY (D.). - Guide du naturaliste dans le Midi de la France. - Tome II. - Neuchâtel, 1963. KEITH (H.G.). - A preliminary check list of Libyan Flora. - London, 1965.

KUNKEL (G.). - The Vegetation of Hormoz, Qeshm and Neighbouring Islands (Persian Gulf Area). - Vaduz, 1977.

LECLERC (L.). - Traité des simples d'Ibn-al-Baytar. - Notices et extraits des manuscrits de la Bibliothèque nationale, XXIII, XXV, XXVI. - Paris, 1877-1883 (3 volumes).

LENORMANT $(F$.$) . - Note sur quelques représentations antiques du Chamaerops humilis. Bulletin de la$ Société botanique de France, $\mathrm{n}^{\circ} 3,1856$, pp. 148-153.

MED-Checklist I / W. Greuter, H. Burdet, G. Lorca Ed. - Genève, 1984.

PACHO (J.R.). - Relation d'un voyage dans la Marmarique, la Cyrénaïque et les oasis d'Audjelah et de Maradeh. - Paris, 1827. POLUNIN (O.), HUXLEY (A.). - Fleurs du bassin méditerranéen (traduction-adaptation de G.G. Aymonin). -
Paris, 1967.

OUÉZEL (P.), SANTA (S.). - Nouvelle flore de I'Algérie et des régions désertiques méridionales. - Tome I. Paris, 1962.

RECHINGER (K.H.). - Flora Iranica. - Graz, 1978 (fascicule 131).

SFIKAS (G.). - Trees and shrubs of Greece. - $2^{\mathrm{e}}$ edition. - Athens, 1981.

STEWART (R.R.). - An annotated Catalogue of the vascular plants of West Pakistan and Kashmir. In: Nasir and Ali. - Flora of West Pakistan. - Karachi, 1972.

TOMLINSON (P.B.). - The Botany of Mangroves. - Cambridge, 1986.

TOURNEFORT (J. PITTON de). - Relation d'un voyage du Levant. - Lyon, 1717. 